\title{
Relationship between hypoalbuminemia, hyperlipidemia and renal severity in patients with lupus nephritis: a prospective study
}

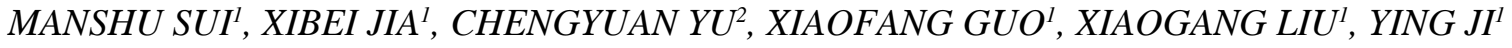 \\ SUHONG MU ${ }^{l}$, HONGCHI WU ${ }^{l}$, RUJUAN XIE ${ }^{l}$
}

Manshu Sui and Xibei Jia contributed equally to this work.

${ }^{1}$ Department of Nephrology, First Affiliated Hospital of Harbin Medical University, Harbin, PR China

${ }^{2}$ Department of Cardiology, First Affiliated Hospital of Harbin Medical University, Harbin, PR China

\begin{abstract}
Aim of the study: The purpose is to evaluate the relationship between hypoalbuminemia, hyperlipidemia, nephrotic and renal severity in patients with lupus nephritis.

Material and methods: Autoantibodies and serological parameters were measured and analyzed in 429 patients with lupus nephritis in a single centre.

Results: The prevalence for anti-dsDNA, anti-nucleosome and anti-histone was higher in the nephrotic syndrome $(N S)$ patients than that in non-NS patients $(p<0.0001$ for all comparisons). The NS patients had a higher proportion of diffuse proliferative renal lesions $(69.05 \%)$ and membranous lesions (68.00\%). Serum total cholesterol and albumin levels were associated with activity and severity of renal disease. The levels of proteinuria and serum albumin were positively correlated with activity and chronicity index ( $p<0.001$ for all correlations). The incidence of a poor renal outcome $(p=0.0461)$ in the NS patients was significantly increased. On the other hand, the remission rate $(p=0.0002)$ was significantly reduced and recurrence rate $(p=0.0027)$ was significantly increased in NS patients.

Conclusions: This paper highlights that nephrotic-range proteinuria, elevated total cholesterol level and decreased serum albumin levels may reflect the activity and severity of renal damage in SLE patients.
\end{abstract}

Key words: hypoalbuminemia, hyperlipidemia, activity, renal damage, systemic lupus erythematosus.

(Centr Eur J Immunol 2014; 39 (2): 243-252)

\section{Introduction}

Lupus nephritis (LN) is manifested in about $40-90 \%$ of all systemic lupus erythematosus (SLE) patients, and is associated with significant morbidity and a poor prognosis $[1,2]$. Double-stranded DNA (dsDNA), nucleosome, histone, as well as more complex antigenic structures resulting from the assembly of histone and non-histone proteins with DNA [3] are recognized by both cellular and humoral constituents of the immune system in SLE patients with and without LN [4-6]. In active lupus renal disease, both the classic and the alternate pathways of the complement cascade are activated, and serum levels of $\mathrm{C} 3$ and $\mathrm{C} 4$ are often depressed [7, 8]. Moreover, the diverse renal pathologic findings in SLE present with a range of clinical renal syndromes, such as asymptomatic hematuria/proteinuria, nephrotic syndrome (NS), or nephritic syndrome. This may be accompanied by varying degrees of hypertension and renal dysfunction $[9,10]$. Furthermore, higher creatinine, low complement levels, thrombocytopenia, anemia, hypertension, and proliferative glomerulonephritis are predictors of end-stage renal disease (ESRD) in LN within multiethnic cohorts [11-13].

The general characteristics of nephrotic syndrome, including heavy proteinuria, hypoalbuminemia, hyperlipidemia and edema, are well recognized. In patients with $\mathrm{LN}$, the NS is generally associated with diffuse (WHO class IV) or membranous glomerulonephritis (WHO class V) [14]. Several reports showed that presence of nephrotic-range proteinuria is associated with poor renal outcomes [9, 11]. Furthermore, to determine whether an elevated serum total cholesterol level in a first-available sample obtained at a SLE clinic is associated with worse renal outcome and mortality in patients with SLE $[11,15]$. Serum albumin reflects disease activity in SLE patients with nephritis [16]. Giv-

Correspondence: Rujuan Xie, Ph.D., Department of Nephrology, 23 Youzheng Street, First Affiliated Hospital of Harbin Medical University, Harbin, 150001, China, tel. +86 451 85555818, e-mail: docter_sms@yeah.net 
en this background, we hypothesized that nephrotic-range proteinuria, elevated total cholesterol level and decreased serum albumin levels may reflect the activity and severity of renal damage in patients with LN. Up to date, however, there have been no previous reports on the relationship between hypoalbuminemia, hyperlipidemia, nephrotic syndrome and exacerbation of renal disease with SLE in China or available data from a large cohort of lupus nephritis with SLE from previous studies due to its rarity.

Including 429 LN patients, of which 214 were diagnosed with nephrotic syndrome, this large retrospective study aimed to analyze the clinical and pathological features and outcome of LN patients who had nephrotic syndrome with those who did not, and to investigate the association between the different pathologic phenotypes and the specific clinical manifestations of the nephrotic syndrome in a single center. Furthermore, major focus of the present research was to find nephrotic-range proteinuria, elevated total cholesterol level and decreased serum albumin levels may reflect the activity and severity of renal damage in LN patients, and the LN patients with NS are more likely to have poor renal outcome.

\section{Material and methods}

\section{Patients}

In this single-center retrospective study, 429 lupus patients with nephritis, diagnosed from 2002 to 2011 in Harbin Medical University First Hospital, were enrolled. All the patients fulfilled the 1997 American College of Rheumatology revised criteria for SLE [17]. The clinical and pathological data of the patients were retrospectively analyzed. Patients were divided into two groups based on the presence (the NS group) or absence (the non-NS group) of nephrotic syndrome. There were 214 lupus patients (192 females, 23 males; median 34 years, inter-quartile range 10-74 years) with evidence of nephrotic syndrome at baseline, defined as proteinuria, with protein $\geq 3.5 \mathrm{~g} / 24 \mathrm{~h}$, serum albumin level $\leq 30 \mathrm{~g} / \mathrm{l}$, and edema. The second group consisted of 215 lupus patients (201 females, 13 males; median 35 years, inter-quartile range $10-80$ years) without clear evidence of nephrotic syndrome. No significant differences were found between the groups for the gender and age. The research was in compliance with the Declaration of Helsinki and approved by the ethics committee of Harbin Medical University First Hospital. Informed consent was obtained from each patient.

\section{Laboratory and serological measures}

24-hour urine protein excretion was measured by an immunoturbidimetry. Serum albumin (Alb), serum total cholesterol (TChol), serum total triglyceride (TG), serum creatinine (Scr) and blood urea nitrogen (BUN) concentration was determined by an automatic analyzer. In addition, autoantibod- ies were detected by the European Union immunoblot assay for all patients. Complement factor 3 (C3, normal range, 0.88 to $2.01 \mathrm{~g} / \mathrm{l})$ and complement factor $4(\mathrm{C} 4$, normal range, 0.10 to $0.47 \mathrm{~g} / \mathrm{l})$ were determined by nephelometry.

\section{Renal histopathology}

Renal biopsy was performed in 150 of the 429 lupus patients with nephritis (75 NS and 75 non-NS patients). Renal specimens were evaluated using light and electron microscopy and direct immunofluorescence. For light microscopy, paraffin sections were stained with silver, periodic acid-Schiff, hematoxylin \& eosin and trichrome and were forwarded to two pathologists. Renal histopathology was classified according to the World Health Organization and International Society of Nephrology/Renal Pathology Society as follows: 16 patients had class I lesions, 76 patients had class II lesions, 7 had class III lesions, 48 had class IV lesions, 30 had class V lesions and one patient had class VI lesions [18].

Pathological parameters such as the activity index (AI) and chronicity index (CI) were approached using a modification of the criteria proposed [19]. Activity index includes endocapillary hypercellularity, leukocyte infiltration, cellular crescents, karyorrhexis, fibrinoid necrosis, formation of wire-loop and interstitial inflammatory cell infiltration. CI includes glomerular sclerosis, fibrous crescents and interstitial fibrosis. Differences in scoring between the two pathologists were resolved by re-reviewing the biopsies and coming to a consensus.

\section{Renal treatment and outcome measures}

The patients were treated in accordance with clinical routine for $\mathrm{LN}$ [20]. The mainstay of induced therapy was prednisone ( $1 \mathrm{mg} / \mathrm{kg}$ per day) combined with cyclophosphamide (Cyc, $0.75 \mathrm{~g} / \mathrm{m}^{2}$ per month). Patients with severe necrotizing crescentic glomerulonephritis and diffuse pulmonary alveolar hemorrhage were also treated with methylprednisone (MP, 7-15 mg/kg per day, 3 days) pulse therapy. For maintenance therapy, a low dose of prednisone combined with immunosuppressive drugs such as azathioprine and mycophenolate mofetil were administered.

The remission of LN includes complete remission and partial remission. Complete remission was defined as urinary protein excretion $<0.3 \mathrm{~g} / \mathrm{day}$, normal urinary sediment (red blood cell $<3 / \mathrm{HP}$, white blood cell $<5 / \mathrm{HP}$ ), normal serum albumin and normal renal function. Partial remission was defined as having any one of the following items: decrease of Scr to $<130 \mu \mathrm{mol} / \mathrm{l}$ for patients with a baseline Scr level $\geq 130 \mu \mathrm{mol} / \mathrm{l}$ but $\leq 260 \mu \mathrm{mol} / \mathrm{l}$; decrease of Scr by $>50 \%$ for patients with a baseline Scr level $>260 \mu \mathrm{mol} / \mathrm{l}$; decrease of urinary protein excretion by $>50 \%$ and $<3.0 \mathrm{~g} /$ day, with a serum albumin level $\geq 30 \mathrm{~g} / \mathrm{l}$ and stable renal function. A poor renal outcome was defined as doubling Scr values for a period of at least six months, 
with a minimum value of Scr of $176.8 \mu \mathrm{mol} / \mathrm{l}$. Renal relapse is doubling of the lowest Scr observed so far and/or developing either NS while the lowest proteinuria had been $<2.0 \mathrm{~g} /$ day repeatedly, or proteinuria $>1.5 \mathrm{~g}$ /day without other causes in a patient who does not have proteinuria.

\section{Statistical analyses}

Differences of quantitative parameters between groups were assessed using the non-parametric test. Differences of qualitative data were compared using the $\chi^{2}$ test or Fisher exact test. The odds ratio (OR) was calculated for assessing the risk of appearance of each variable. A lower limit of the $95 \%$ confidence interval $(\mathrm{CI})$ that exceeded 1.0 was taken to indicate statistical significance in the case of positive association and an upper limit lower than 1.0 in the case of negative association. The Spearman Correlation was used to analyze correlation. Analysis was performed with statistical software GraphPad Prism (version 5.0; GraphPad Software, Inc., San Diego, CA, USA). $P<0.05$ is considered statistically significant.

\section{Results}

The nephrotic syndrome patients suffered more severe renal disease damage than non-nephrotic syndrome patients

To study the clinical characteristics of NS, the groups of NS $(n=214)$ and non-NS $(n=215)$ patients in the total of $429 \mathrm{LN}$ patients were compared with respect to related clinical and serological parameters. First of all, the age and gender distribution was balanced between NS and non-NS groups (Table 1). The average levels of 24-hour urine protein excretion, urinary RBC, Scr and BUN was significantly higher in NS patients than those in non-NS patients $(p<0.001$ for all comparisons), while the average levels of platelet (PLT), hemoglobin (HB), serum C3 and C4 were significantly lower in the NS group than those in the nonNS group ( $p<0.0001$ for all comparisons) (Table 1$)$. No significant association was found between NS and other laboratory parameters (Table 1).

\section{Prevalence of three autoantibody reactivity in lupus nephritis patients with nephrotic syndrome}

Table 2 summarizes the findings of the autoantibody profile in LN patients with/out NS. Significantly, prevalence of three out of the sixteen routinely tested antibodies in our center was observed in LN patients with NS as compared to those without NS. Of the 214 patients with NS, 73.83\% (158) of anti-dsDNA (OR $=2.848$, $p<0.0001), 67.29 \%$ (144) of anti-nucleosome (OR $=2.699$, $p<0.0001)$ and $59.81 \%$ (128) of anti-histone (OR = $=2.276, p<0.0001)$ were positive. Positive rates of other tested autoantibodies such as anti-RNP, anti-Ro, anti-Sm and anti-centromere were not associated with NS in this group. Furthermore, the positive rates among the respec-

Table 1. Comparisons of clinical parameters between NS and non-NS patients with LN

\begin{tabular}{lccc}
\hline Index & NS group $(\boldsymbol{n}=\mathbf{2 1 4})$ & Non-NS group $(\boldsymbol{n}=\mathbf{2 1 5})$ & $\boldsymbol{p}$ \\
\hline Age $(\mathrm{yr})$ & $35(10-80)$ & $34(10-74)$ & 0.9988 \\
\hline Female & $201(93.93)$ & $192(89.30)$ & 0.1161 \\
\hline Proteinuria $(\mathrm{g} / 24 \mathrm{~h})$ & $4.44(0.95-16.08)$ & $0.97(0.36-5.55)$ & $<\mathbf{0 . 0 0 0 1}$ \\
\hline Hematuria $(\mathrm{n} / \mathrm{hpf})$ & $9.13(0-879.1)$ & $2.48(0.07-195.2)$ & $<\mathbf{0 . 0 0 0 1}$ \\
\hline Scr $(\mu \mathrm{mol} / \mathrm{l})$ & $76.3(31.8-665)$ & $64.3(30.6-978)$ & $<\mathbf{0 . 0 0 0 1}$ \\
\hline BUN $(\mathrm{mmol} / \mathrm{l})$ & $7.45(1.60-54.47)$ & $5.16(2.00-47.66)$ & $<\mathbf{0 . 0 0 0 1}$ \\
\hline WBC $\left(\times 10^{9} / \mathrm{l}\right)$ & $4.23(0.39-23.60)$ & $4.58(1.02-30.40)$ & 0.1184 \\
\hline PLT $\left(\times 10^{9} / \mathrm{l}\right)$ & $86.25(4.4-385.0)$ & $169.0(2.00-584)$ & $<\mathbf{0 . 0 0 0 1}$ \\
\hline HB $(\mathrm{g} / \mathrm{l})$ & $90.50(26.20-148)$ & $0.58(0.17-1.86)$ & $<\mathbf{0 . 0 0 0 1}$ \\
\hline C3 $(\mathrm{g} / \mathrm{l})$ & $0.38(0.06-1.55)$ & $0.11(0.01-0.63)$ & $<\mathbf{0 . 0 0 0 1}$ \\
\hline C4 $(\mathrm{g} / \mathrm{l})$ & $0.07(0.01-0.43)$ & $5(1-14)$ & $<\mathbf{0 . 0 0 0 1}$ \\
\hline AI $($ out of 24$)$ & $7(1-18)$ & $1(0-9)$ & $\mathbf{0 . 0 0 0 1}$ \\
\hline CI $($ out of 12) & $2(0-8)$ & $\mathbf{0 . 0 0 3 6}$ \\
\hline
\end{tabular}

Data of 429 patients with LN. Values are shown as medians (IQR) or numbers (\%)

NS - nephrotic syndrome; IQR - inter-quartile range. Scr - serum creatinine; BUN-blood urea nitrogen; TChol-total cholesterol; TG - total triglycerides; $W B C$ - white blood cell count; PLT - platelet count; HB - hemoglobin; C3-complement factor 3; C4 - complement factor 4 $p<0.05$ indicates a difference; $p<0.01$ indicates a significant difference. 
tive three antibodies including anti-dsDNA, anti-nucleosome and anti-histone in NS and non-NS patients with SLE were comparable $(73.83 \%, 67.29 \%$ and $59.81 \%$ in NS group; $49.77 \%, 43.26 \%$ and $39.53 \%$ in non-NS group, Table 2).

\section{Hypercholesterolemia and hypoproteinemia may reflect the activity and severity of renal damage in lupus nephritis patients with nephrotic syndrome}

We further analyzed the clinical relevance of hypercholesterolemia and hypoproteinemia as a marker of the activity and severity of renal damage in 214 NS patients. Among these patients, hypercholesterolemia was significantly correlated with higher levels of proteinuria ( $p$ $<0.0001)$, Scr $(p=0.0330)$, BUN $(p=0.0021)$ and serum TG $(p<0.0001)$, while we also found that there was a significant correlation between hypercholesterolemia and lower levels of serum albumin $(p<0.0001)$ and $\mathrm{C} 4$ $(p=0.0449)$ (Table 3). However, no significant association was found between hypercholesterolemia and other laboratory parameters (Table 3). On the other hand, pa- tients with serum albumin levels $\leq 20 \mathrm{~g} / \mathrm{l}$ were younger $(p=0.0151)$ than patients with serum albumin levels $>$ $20 \mathrm{~g} / \mathrm{l}$. Furthermore, we also found that there was a strong positive correlation between serum albumin levels and higher levels of proteinuria $(p<0.0001)$, serum TChol $(p<0.0001)$ and TG $(p=0.0007)$ (Table 4$)$.

\section{The nephrotic syndrome tended to suffer from/ be presented/related with diffuse proliferative or membranous glomerulonephritis in lupus nephritis patients}

Analysis of the histological classification of NS revealed a higher frequency of diffuse proliferative glomerulonephritis (class IV) $(69.05 \%$ vs. $34.94 \%, p=0.0005)$ and membranous lesions (class V) $(68.00 \%$ vs. $34.94 \%, p=$ $=0.0030)$ compared to non-diffuse proliferative renal lesions (class I + II + III), respectively in the same patient group. There was no significant difference between class IV and class V (69.05\% vs. $68.00 \%, p=1.0000)$ (Fig. 1A). On the other hand, the prevalence of hypercholesterolemia in patients with diffuse proliferative renal lesions (class IV) was significantly higher than that in patients with

Table 2. Prevalence of autoantibodies in patients with LN

\begin{tabular}{|c|c|c|c|c|}
\hline Antibodies & NS $(n=214)$ & Non-NS $(n=215)$ & OR $(95 \% \mathrm{CI})$ & $P$ \\
\hline ANA & $206(96.26)$ & $204(94.88)$ & $1.388(0.5471-3.524)$ & 0.6400 \\
\hline Anti-mitochondria & 25 (11.68) & $16(7.44)$ & $1.645(0.8516-3.178)$ & 0.1430 \\
\hline Anti-ribosome & 47 (21.96) & $63(29.30)$ & $0.679(0.4386-1.051)$ & 0.0971 \\
\hline Anti-histone & $128(59.81)$ & $85(39.53)$ & $2.276(1.5460-3.351)$ & $<0.0001$ \\
\hline Anti-nucleosome & $144(67.29)$ & $93(43.26)$ & $2.699(1.822-3.998)$ & $<0.0001$ \\
\hline Anti-dsDNA & $158(73.83)$ & 107 (49.77) & $2.848(1.898-4.272)$ & $<0.0001$ \\
\hline Anti-PNCA & $7(3.27)$ & $4(1.86)$ & $1.784(0.5143-6.187)$ & 0.3808 \\
\hline Anti-centromere & $5(2.34)$ & $5(2.33)$ & $1.005(0.2866-3.523)$ & 1.0000 \\
\hline Anti-Jo1 & 0 & 0 & undefined & undefined \\
\hline Anti-PM-Scl & $3(1.40)$ & $1(0.47)$ & $3.043(0.3138-29.50)$ & 0.3721 \\
\hline Anti-Scl70 & $4(1.87)$ & $7(3.26)$ & $0.566(0.1632-1.963)$ & 0.5435 \\
\hline Anti-SSB & 37 (17.29) & $36(16.74)$ & $1.039(0.6280-1.720)$ & 0.8984 \\
\hline Anti-Ro52 & $103(48.13)$ & $102(47.44)$ & $1.028(0.7037-1.502)$ & 0.9231 \\
\hline Anti-SSA & $129(60.28)$ & $124(57.67)$ & $1.114(0.7578-1.637)$ & 0.6239 \\
\hline Anti-Sm & $49(22.90)$ & $49(22.79)$ & $1.006(0.6409-1.579)$ & 1.0000 \\
\hline Anti-RNP & $63(29.44)$ & $80(37.21)$ & $0.704(0.4702-1.054)$ & 0.1013 \\
\hline \multicolumn{5}{|c|}{$\begin{array}{l}\text { Values are shown as numbers }(\%) \\
L N-\text { lupus nephritis; } N S-n e p h r o t i c \text { syndrome; OR - odds ratio; ANA - antinuclear antibody; anti-dsDNA - anti-double-stranded DNA antibody; anti-PNCA - } \\
\text { anti-proliferative cell nuclear antigen; anti-Sm - anti-Smith antibody; anti-SSA - anti-Sjögren's syndrome antigen A; anti-SSB - anti-Sjögren's syndrome antigen } \\
B ; \text { anti-RNP - anti-ribonucleoprotein; anti-RibP-anti-ribosomal P protein antibody } \\
\text { Prevalence of three antibodies tested was significantly increased in LN patients with nephrotic syndrome as compared to patients without nephrotic syndrome } \\
p<0.05 \text { indicates a difference; } p<0.01 \text { indicates a significant difference. }\end{array}$} \\
\hline
\end{tabular}


Table 3. Demographic and clinical features of patients with nephrotic syndrome according to the serum total cholesterol levels

\begin{tabular}{lccc}
\hline Index & With hypercholesterolemia $(\boldsymbol{n}=\mathbf{8 7})$ & Without hypercholesterolemia $(\boldsymbol{n}=\mathbf{1 2 7})$ & $\boldsymbol{p}$ \\
\hline Age $>45 \mathrm{yr}$ & $12(24.04)$ & $27(21.26)$ & 0.2075 \\
\hline Female & $84(96.55)$ & $117(92.13)$ & 0.2483 \\
\hline Proteinuria $>6.0 \mathrm{~g} / 24 \mathrm{~h}$ & $41(41.38)$ & $22(17.32)$ & $<\mathbf{0 . 0 0 0 1}$ \\
\hline Hematuria $>3 \mathrm{n} / \mathrm{hpf}$ & $58(58.62)$ & $79(62.20)$ & 0.5629 \\
\hline Scr $>133 \mu \mathrm{mol} / 1$ & $27(31.03)$ & $23(18.11)$ & $\mathbf{0 . 0 3 3 0}$ \\
\hline BUN $>7.1 \mathrm{mmol} / 1$ & $56(56.32)$ & $54(42.52)$ & $\mathbf{0 . 0 0 2 1}$ \\
\hline TG $>1.71 \mathrm{mmol} / 1$ & $76(75.86)$ & $77(60.63)$ & $<\mathbf{0 . 0 0 0 1}$ \\
\hline Alb $\leq 20 \mathrm{~g} / 1$ & $41(41.38)$ & $14(11.02)$ & $<\mathbf{0 . 0 0 0 1}$ \\
\hline WBC $<4 \times 10^{9} / 1$ & $37(42.53)$ & $67(52.76)$ & 0.1645 \\
\hline PLT $\leq 100 \times 10^{9} / 1$ & $23(26.44)$ & $48(37.80)$ & 0.1039 \\
\hline HB $\leq 110 \mathrm{~g} / 1$ & $64(73.56)$ & $105(82.68)$ & 0.1254 \\
\hline C $\leq 0.88 \mathrm{~g} / 1$ & $76(87.36)$ & $117(92.13)$ & 0.2540 \\
\hline $\mathrm{C} 4 \leq 0.10 \mathrm{~g} / 1$ & $69(79.31)$ & $84(66.14)$ & $\mathbf{0 . 0 4 4 9}$ \\
\hline
\end{tabular}

Values are shown as numbers (\%)

Alb-albumin; Scr-serum creatinine; BUN-blood urea nitrogen; TChol-total cholesterol; TG - total triglycerides; WBC-white blood cell count; PLT-platelet count; $\mathrm{HB}$ - hemoglobin; $\mathrm{C} 3$ - complement factor 3; C4 - complement factor 4

$p<0.05$ indicates a difference; $p<0.01$ indicates a significant difference

Table 4. Demographic and clinical features of patients with nephrotic syndrome according to the serum albumin levels

\begin{tabular}{lccc}
\hline Index & Serum Alb $\leq \mathbf{2 0}$ g/l $(\boldsymbol{n}=\mathbf{5 7})$ & Serum Alb $>\mathbf{2 0}$ g/l $(\boldsymbol{n}=\mathbf{1 5 7})$ & $\boldsymbol{p}$ \\
\hline Age $>45 \mathrm{gr}$ & $7(12.28)$ & $41(26.11)$ & $\mathbf{0 . 0 1 5 1}$ \\
\hline Female & $53(92.98)$ & $148(94.27)$ & 0.7495 \\
\hline Proteinuria $>6.0 \mathrm{~g} / 24 \mathrm{~h}$ & $48(84.21)$ & $16(10.19)$ & $<\mathbf{0 . 0 0 0 1}$ \\
\hline Hematuria $>3 \mathrm{n} / \mathrm{hpf}$ & $42(73.68)$ & $97(61.78)$ & 0.1442 \\
\hline Scr $>133 \mu \mathrm{mol} / 1$ & $14(24.56)$ & $33(21.02)$ & 0.5796 \\
\hline BUN $>7.1 \mathrm{mmol} / 1$ & $35(61.40)$ & $75(47.77)$ & 0.0897 \\
\hline TChol $>5.7 \mathrm{mmol} / 1$ & $44(77.19)$ & $42(26.75)$ & $<\mathbf{0 . 0 0 0 1}$ \\
\hline TG $>1.71 \mathrm{mmol} / 1$ & $50(87.72)$ & $104(66.24)$ & $<\mathbf{0 . 0 0 0 1}$ \\
\hline WBC $<4 \times 10^{9} / 1$ & $28(49.12)$ & $72(45.86)$ & 0.7570 \\
\hline PLT $\leq 100 \times 10^{9} / 1$ & $20(35.09)$ & $56(35.67)$ & 1.0000 \\
\hline HB $\leq 110 \mathrm{~g} / 1$ & $41(71.93)$ & $127(80.89)$ & 0.1879 \\
\hline C $\leq 0.88 \mathrm{~g} / 1$ & $51(89.47)$ & $141(89.81)$ & 1.0000 \\
\hline C4 $\leq 0.10 \mathrm{~g} / 1$ & $48(84.21)$ & $119(75.80)$ & 0.1463 \\
\hline
\end{tabular}

Values are shown as numbers (\%)

Alb-albumin; Scr-serum creatinine; BUN-blood urea nitrogen; TChol-total cholesterol; TG-total triglycerides; WBC-white blood cell count; PLT-platelet count; HB - hemoglobin; $C 3$ - complement factor 3; C4 - complement factor 4

$p<0.05$ indicates a difference; $p<0.01$ indicates a significant difference 
non-diffuse proliferative lesions (class I + II + III) $(69.57 \%$ vs. $28.00 \%, p=0.0085$ ) (Fig. 1B), while the prevalence of serum albumin levels $\leq 20 \mathrm{~g} / 1$ in patients with membranous lesions (class $\mathrm{V}$ ) was significantly higher than that in patients with non-diffuse proliferative lesions (class I + II + III, $64.71 \%$ vs. $17.24 \%, p=0.0003$ ) and diffuse proliferative renal lesions (class IV, $64.71 \%$ vs. $31.03 \%$, $p=0.0348$ ) (Fig. 1C).

For the pathological features, we found that average AI $(p=0.0001)$ and CI $(p=0.0036)$ scores in LN patients with NS were significantly higher than in non-NS patients (Table 1). Meanwhile, we found that $\mathrm{LN}$ patients with NS presented with a distinguished high incidence of crescent $(36.00 \%$ vs. $8 \%, p<0.0001)$, tubular atrophy $(60.00 \%$ vs. $38.00 \%, p=0.0003)$ and interstitial fibrosis $(62.67 \%$ vs. $41.33 \%, p=0.0140)$ (Table 5). On the other hand, there was no significant difference in the occurrence of segmental necrosis, wire-loops, glomerulosclerosis and vascular lesion between the two groups. Significantly, we also found that there was a strong positive correlation between the levels of proteinuria and AI $(r=0.3495, p<$ $0.0001)$ and CI $(r=0.3568, p<0.0001)$ (Figs. 2A and 2B). A negative correlation was found between the levels of serum albumin and $\mathrm{AI}(r=-0.3719, p<0.0001)$ and $\mathrm{CI}$ $(r=-0.3005, p=0.0002)$ (Figs. 2C and 2D).

A

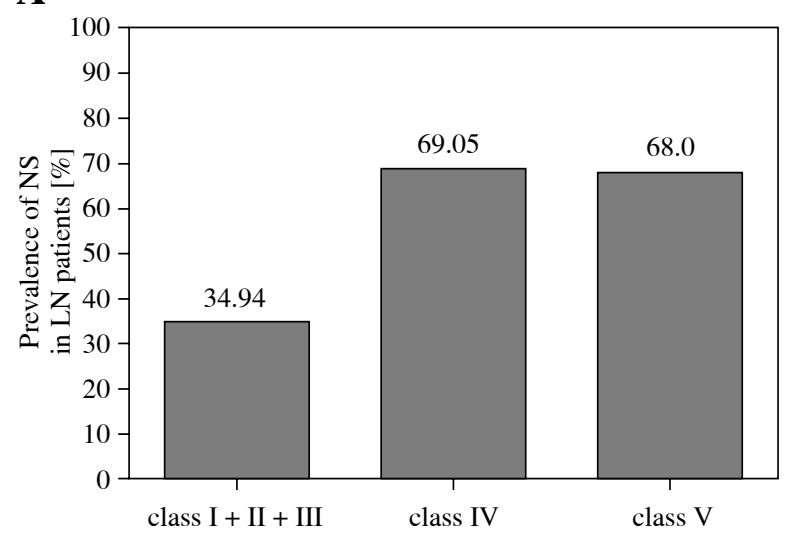

C

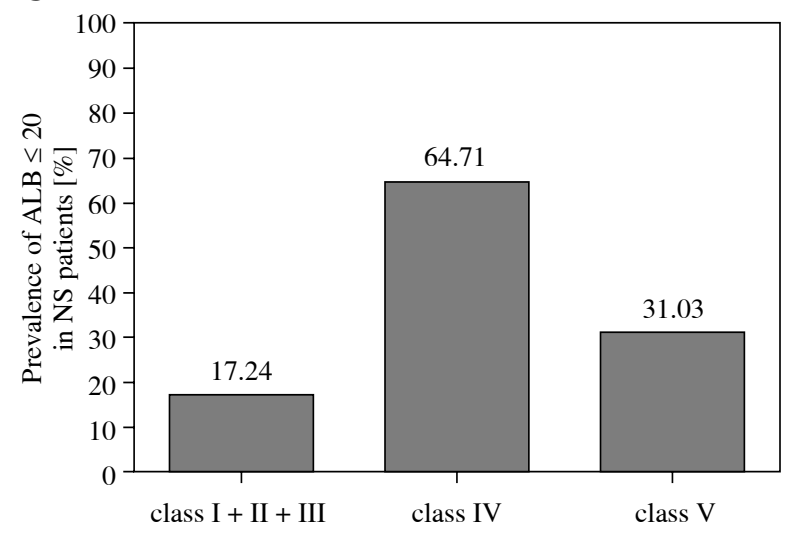

\section{The NS patients had worse outcomes than non- nephrotic syndrome within the lupus nephritis patient group}

General treatment protocols were comparable between NS and non-NS patients in the LN group of patients. Of the $429 \mathrm{LN}$ patients, a total of 35 patients, including 11 NS and 24 non-NS patients, were treated with oral corticosteroids alone ( $p=0.0330)$. Comparably, 167 of 215 non-NS patients and 179 of 214 NS patients received corticosteroids combined with cyclophosphamide ( $p=0.1423$ ). However, a significant difference was obtained between the two patient groups who had to take intravenous methylprednisolone pulse therapy (82/214 for NS vs. 36/215 for non-NS patients, $p<0.0001$, Table 6). Furthermore, the total remission rate including both partial and complete remission in NS patients was significantly lower than that in non-NS patients based on a mean follow-up time of 13 months (range 10 to 14 months; $76.64 \%$ vs. $90.23 \%$, $p=0.0245$, Table 6). Notably, NS patients tended to have worse renal disease outcome than non-NS, as defined by doubling the serum creatinine value for a period of at least 6 months, with a minimum value of serum creatinine of $176.8 \mu \mathrm{mol} / \mathrm{l}(6.98 \%$ vs. $2.79 \%, p=0.0461)$. In addition, at their respective last observations before they were dis-

B

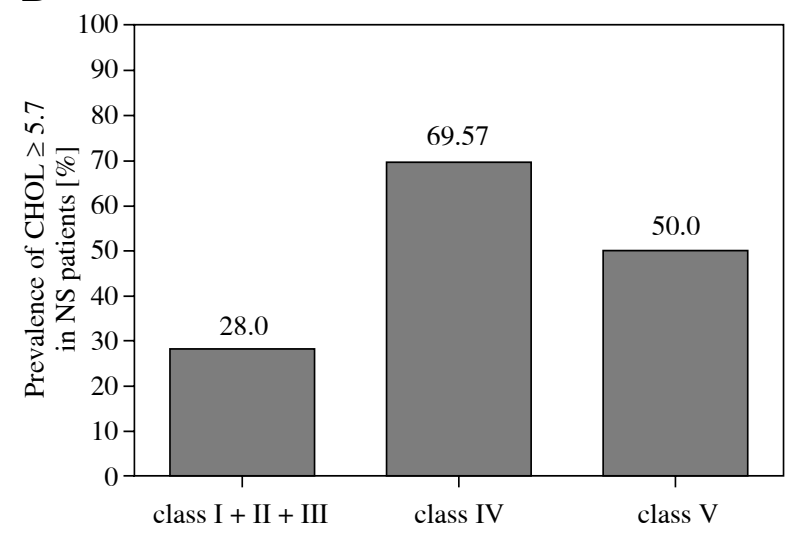

Fig. 1. A) Prevalence of NS in patients with diffuse proliferative renal lesions (class IV) and membranous lesions (class V) versus non-diffuse proliferative lesions (class I + II + III) $(p=0.0005, p=0.0023$, respectively); B) Prevalence of $\mathrm{CHOL} \geq 5.7$ in patients with diffuse proliferative renal lesions (class IV) versus non-diffuse proliferative lesions (class I + II + III) and membranous lesions (class V) ( $p=0.0085, p=0.3035$, respectively). C) Prevalence of $\mathrm{ALB} \leq 20$ in patients with membranous lesions (class V) versus non-diffuse proliferative lesions (class I + II + III) and diffuse proliferative renal lesions (class IV) $(p=$ $=0.0003, P=0.0348$, respectively) 
Table 5. Correlations between nephrotic syndrome and the relevant histopathologic parameters

\begin{tabular}{lccc}
\hline Histopathologic parameters & NS $(\boldsymbol{n}=\mathbf{7 5})$ & Non-NS $(\boldsymbol{n}=\mathbf{7 5})$ & $\boldsymbol{P}$ \\
\hline Crescent & $27(36.00)$ & $6(8.00)$ & $<\mathbf{0 . 0 0 0 1}$ \\
\hline Segmental necrosis & $13(17.33)$ & $8(10.67)$ & 0.3469 \\
\hline Wire-loops & $13(17.33)$ & $11(14.67)$ & 0.8242 \\
\hline Glomerulosclerosis & $23(30.67)$ & $18(24.00)$ & 0.3678 \\
\hline Tubular atrophy & $60(80.00)$ & $38(50.67)$ & $\mathbf{0 . 0 0 0 3}$ \\
\hline Interstitial fibrosis & $47(62.67)$ & $31(41.33)$ & $\mathbf{0 . 0 1 4 0}$ \\
\hline Vascular lesion & $22(29.33)$ & $13(17.33)$ & 0.1218 \\
\hline Activity index, score & $7(1-18)$ & $5(1-14)$ & $\mathbf{0 . 0 0 0 1}$ \\
\hline Chronicity index, score & $2(0-8)$ & $1(0-9)$ & $\mathbf{0 . 0 0 3 6}$ \\
\hline
\end{tabular}

Values were show as number $(\%)$ or median (IQR). IQR, inter-quartile range. $P<0.05$ indicates difference; $p<0.01$ indicates significant difference.

A

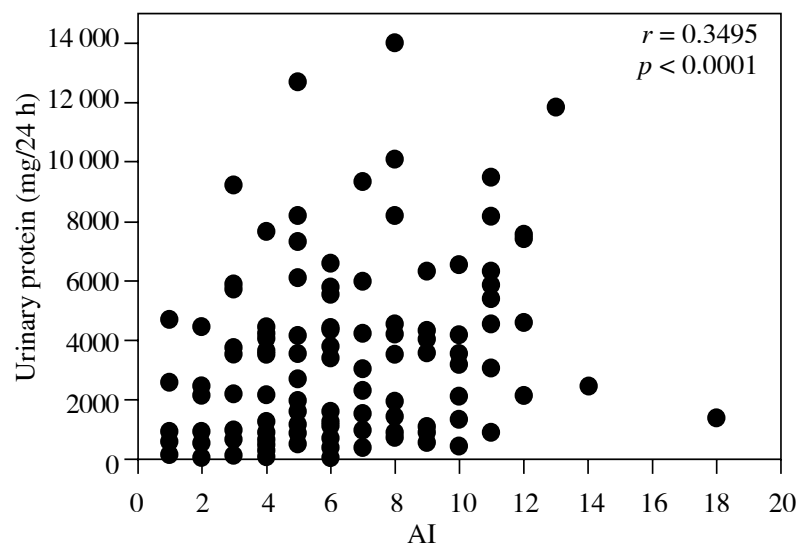

C

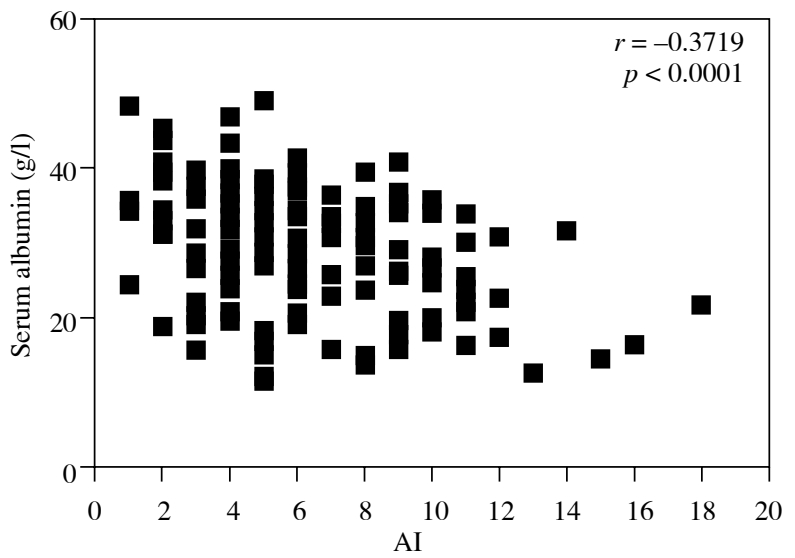

B

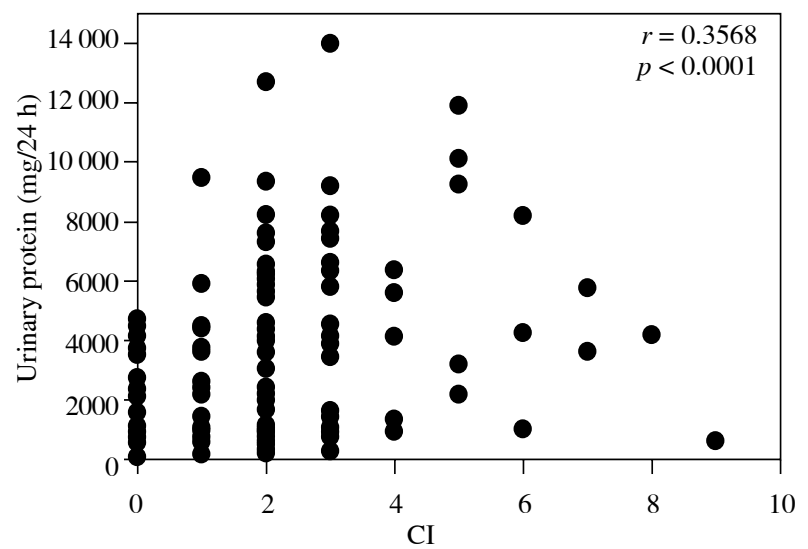

D

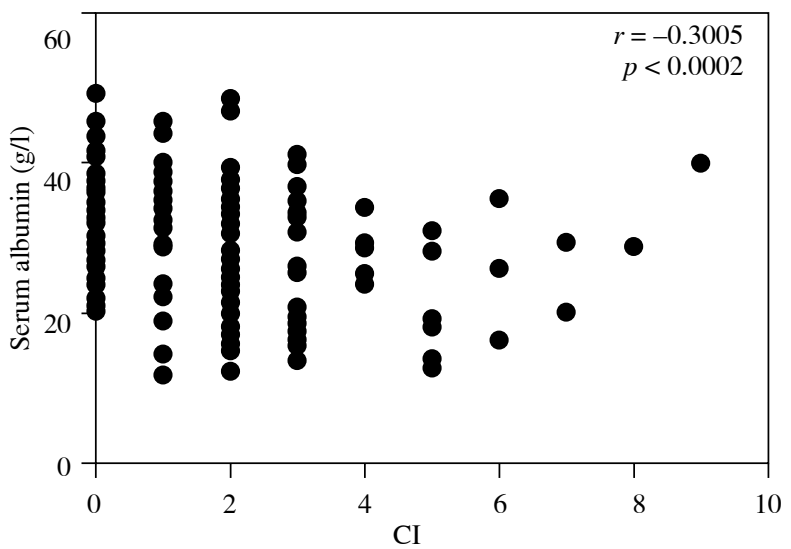

Fig. 2. Correlation between proteinuria / serum albumin level / and activity index (AI) / chronicity index (CI) in patients with lupus nephritis. A) Correlation between proteinuria and AI $(r=0.3495, p<0.0001)$; B) Correlation between serum albumin level and CI $(r=0.3586, p<0.0001)$; C) Correlation between serum albumin level and AI $(r=-0.3719, p<$ $0.0001)$; D) Correlation between serum albumin level and CI $(r=-0.3005, p=0.0002)$ 
Table 6. Clinical status of the patients at their last observation

\begin{tabular}{lccc}
\hline Index & NS group $(\boldsymbol{n}=\mathbf{2 1 4})$ & Non-NS group $(\boldsymbol{n = 2 1 5})$ & $\boldsymbol{P}$ \\
\hline Oral corticosteroid alone & $11 / 214(5.14)$ & $24 / 215(11.16)$ & $\mathbf{0 . 0 3 3 0}$ \\
\hline Corticosteroid combined CTX & $179 / 214(83.64)$ & $167 / 215(77.67)$ & 0.1423 \\
\hline Methylprednisolone pulse therapy & $82 / 214(38.32)$ & $36 / 215(16.74)$ & $<\mathbf{0 . 0 0 0 1}$ \\
\hline Remission rates & $164 / 214(76.64)$ & $194 / 215(90.23)$ & 0.0002 \\
\hline Patients with poor outcome & $15 / 214(6.98)$ & $6 / 215(2.79)$ & 0.0461 \\
\hline Plasma creatinine at last observation $(\mu \mathrm{mol} / \mathrm{l})$ & $69.30(17.00-976.0)$ & $67.95(43.40-652.2)$ & 0.9236 \\
\hline Proteinuria at last observation $(\mathrm{g} / 24 \mathrm{~h})$ & $1.05(0.36-8.08)$ & $0.26(0.19-4.87)$ & $<\mathbf{0 . 0 0 0 1}$ \\
\hline Mortality rates & $6 / 214(2.80)$ & $3 / 215(1.40)$ & 0.3380 \\
\hline ESRD rates & $7 / 214(3.27)$ & $3 / 215(1.40)$ & 0.2205 \\
\hline Recurrence rate & $87 / 172(50.58)$ & $54 / 159(33.96)$ & $\mathbf{0 . 0 0 2 7}$
\end{tabular}

Values were show as median (IQR) or number (\%). IQR, inter-quartile range. Poor outcome: defined as a doubling in serum creatinine values for a period of at least 6 months, with a plasma creatinine value of at least $176.8 \mu \mathrm{mol} / \mathrm{l}$. ESRD, end-stage renal disease. $P<0.05$ indicates difference; $P<0.01$ indicates significant difference

charged from the hospital, NS patients had a significantly higher average level of proteinuria (1.05 vs. $0.26 \mathrm{~g} / 24 \mathrm{~h}$, $p<0.0001)$ than non-NS patients. For the total of $429 \mathrm{LN}$ patients, although respective rates of the worst disease outcome including mortality and ESRD (progression to end-stage renal disease) were not significantly different between NS and non-NS groups (Table 6), after a mean follow-up time of 25 months (range 22 to 27 months) post-treatment, the recurrence rate in the NS group was significantly higher than that in the non-NS group $(50.58 \%$ vs. $33.96 \%, p=0.0027$ ).

\section{Discussion}

In this study, we retrospectively analyzed the clinical feature and outcome of $429 \mathrm{LN}$ patients. Prevalence of anti-dsDNA, anti-nucleosome and anti-histone positivity was more common in LN patients with nephrotic syndrome (NS), with very high specificity. Notably, serum total cholesterol and albumin levels are associated with the activity and severity of renal damage in LN patients with NS. The kidney biopsy showed that more than $50 \%$ was nephrotic syndrome in diffuse proliferative renal lesions (class IV) and membranous lesions (class V). Furthermore, proteinuria and serum albumin levels have a strong positive correlation with $\mathrm{AI}$ and $\mathrm{CI}$ in LN patients with NS. Finally, LN patients with NS had significantly worse renal disease outcomes, lower remission and higher recurrence rates than patients without NS during a long-term follow-up.

The current study first analyzed differences of demographic and clinical features between the NS and non-NS patients. Our findings have shown that 214 (49.88\%) patients had nephrotic syndrome, which was different from the previous reports $[21,22]$. The differing prevalence of nephrotic syndrome among the different populations studied might reflect the relative frequency of class IV and class V among each population. Furthermore, it was found that LN patients with NS had a significantly higher level of urinary RBC, Scr and BUN, and had a lower level of PLT, HB, serum C3 and C4 than those in non-NS patients. More importantly, the distribution of pathological types is largely different between NS and non-NS patients with LN. Patients with NS had a higher proportion of diffuse proliferative renal lesions (class IV) and membranous lesions (class V) and a lower proportion of non-diffuse proliferative renal lesions (class I + II + III), in accordance with previous studies [14]. However, this was different from the results found in a study by Williams et al. [23]. Williams et al. reported that nephrotic range proteinuria was most common in classes III and IV. The discrepancy of positive prevalence might be explained by various subjects enrolled from different races. Therefore, the selection bias could not be excluded.

It was reported that over 150 autoantibodies [24] had been identified in sera of patients with SLE; however, only a few of them were associated with the development of LN. Our findings have shown that serum anti-dsDNA, anti-nucleosome and anti-histone positivity was more common in LN patients with NS, with very high specificity. This is consistent with most of the previously described reports but did have any different prevalence [25-27]. The discrepancy of positive prevalence might be explained by various subjects enrolled from different races, and different assays used to detect these antibodies. Notably, previously reports have indicated only that the prevalence of these autoantibodies was higher in LN, but the difference between the NS and non-NS patients 
was not investigated. This could indicate that positivity of anti-dsDNA, anti-nucleosome and anti-histone might be an available parameter to predict disease activity in LN patients with NS.

The most important finding in this study was the association between serum total cholesterol levels and albumin and the activity and severity of renal damage in LN patients with NS. In this study, the total cholesterol level was associated with proteinuria, Scr, serum albumin levels, serum triglyceride and $\mathrm{C} 4$, in accordance with previous studies $[11,15]$. Indeed, hyperlipidemia can affect the renal function, increase proteinuria and speed up glomerulosclerosis, thus determining a higher risk of progression to dialysis. Furthermore, the persistent proteinuria and dyslipidemia are the major factors for chronic kidney disease progression in SLE patients with renal involvement. In addition, several factors may affect the serum albumin level. However, in our study, all patients had a BMI > 18 and therefore a low serum albumin level due to malnutrition was unlikely. Furthermore, LN may lead to nephrotic range proteinuria, which in turn lowers serum albumin levels. This rationale has prompted clinicians to use serum albumin to indirectly assess activity of LN and conduct its therapy. Yip et al. demonstrated that serum albumin is inversely associated with SLE disease activity and this association was stronger in those with LN and especially in those with proteinuria 16]. Our results support this but we also found that patients with hypoalbuminemia $(\leq 20 \mathrm{~g} / \mathrm{l})$ were significantly younger, and had a higher level of proteinuria, urinary RBC, serum total cholesterol and triglyceride than those without hypoalbuminemia (> $20 \mathrm{~g} / \mathrm{l}$ ). More importantly, this is the first report demonstrating that the prevalence of hypercholesterolemia in patients with diffuse proliferative renal lesions (class IV) was significantly higher, meanwhile the prevalence of hypoalbuminemia $(\leq 20 \mathrm{~g} / \mathrm{l})$ in patients with membranous lesions (class V) was significantly lower/ higher. In addition, our findings have shown that the serum albumin level was more significantly associated with $\mathrm{AI}$ and CI in LN patients with NS. At the same time, we also found that the LN patients with NS presented with a distinguished high incidence of crescent, tubular atrophy and interstitial fibrosis. There are findings to indicate that an elevated serum total cholesterol level and depressed serum albumin levels may reflect the activity and severity of renal damage.

Our data demonstrated that the renal injury in patients with LN seemed to be a more "fulminant" process in NS patients than that in non-NS ones. A number of studies have also reported that nephritic flares were significantly associated with a poor renal outcome $[9,28]$. Results of our study support this finding and also detected a correlation between outcome and nephrotic syndrome. Our study further found a correlation between nephrotic syndrome and the plasma creatinine, as well as proteinuria in the last ob- servation of the LN patients with NS. Moreover, the present study indicated that the nephrotic syndrome is significantly related with reduced remission, increased recurrence rates and poor outcome of renal disease in LN patients, and it is consistent with a previous study [9]. The mortality rates and ESRD rates showed no significant difference between the two subgroups of patients, which contradicts previous studies $[11,29]$. The reasons for the discrepancies across studies are multifactorial, including differences in study design, ethnicity, baseline clinical characteristics and renal parameters, as well as treatment protocols employed. The resulting probability of a poor renal outcome was found to be higher for LN patients with NS.

Finally, it is worth pointing out that this study has several limitations. First, it has inherent flaws of a retrospective study. Only associations can be made by using statistical techniques. Second, the expertise of different clinicians involved in the treatment of these patients differed and this could have an impact on the outcome. Nevertheless, we are convinced that the results obtained provide useful indications that could be further explored in a prospective study on the significance of nephrotic syndrome in LN.

\section{Conclusions}

In summary, the LN patients with NS have a higher proportion of diffuse proliferative renal lesions (class IV) and membranous lesions (class V), serum anti-dsDNA, anti-nucleosome and anti-histone positivity are more common in LN patients with NS. Furthermore, serum total cholesterol and albumin levels are associated with the activity and severity of renal damage in $\mathrm{LN}$ patients with NS. Our study also confirmed that the incidence of a poor renal outcome is significantly increased in LN patients with NS. Therefore, our data suggest the ability of the nephrotic-range proteinuria, elevated total cholesterol level and decreased serum albumin levels to predict renal disease activity, may lead to a major additional benefit in the follow-up of these patients.

We are very grateful to Ms. Jin Xiaoming and Ms. Zhao Ruibo for assistance in the scoring and collecting pathological data of the patients. Furthermore, we also thank Dr. Yu Jianfeng for the excellent English language support.

The authors declare no conflict of interest.

The presented work was supported by the National Natural Science Foundation of China (Grant 81370831).

\section{References}

1. Jakes RW, Bae SC, Louthrenoo W, et al. (2012): Systematic review of the epidemiology of systemic lupus erythematosus in the Asia-Pacific region: prevalence, incidence, clini- 
cal features, and mortality. Arthritis Care Res (Hoboken) 64: 159-168.

2. Osio-Salido E, Manapat-Reyes H (2010): Epidemiology of systemic lupus erythematosus in Asia. Lupus 19: 1365-1373.

3. Burlingame RW, Cervera R (2002): Anti-chromatin (anti-nucleosome) autoantibodies. Autoimmun Rev 1: 321-328.

4. Hirabayashi Y, Oka Y, Tada M, et al. (2007): A potential trigger of nephritogenic anti-DNA antibodies in lupus nephritis. Ann N Y Acad Sci 1108: 92-95.

5. Mortensen ES, Rekvig OP (2009): Nephritogenic potential of anti-DNA antibodies against necrotic nucleosomes. J Am Soc Nephrol 20: 696-704.

6. Sui M, Lin Q, Xu Z, et al. (2013): Simultaneous positivity for anti-DNA, anti-nucleosome and anti-histone antibodies is a marker for more severe lupus nephritis. J Clin Immunol 33: 378-387.

7. Moroni G, Doria A, Ponticelli C (2009): Cyclosporine (CsA) in lupus nephritis: assessing the evidence. Nephrol Dial Transplant 24: 15-20.

8. Birmingham DJ, Irshaid F, Nagaraja HN, et al. (2010): The complex nature of serum $\mathrm{C} 3$ and $\mathrm{C} 4$ as biomarkers of lupus renal flare. Lupus 19: 1272-1280.

9. Contreras G, Pardo V, Cely C, et al. (2005): Factors associated with poor outcomes in patients with lupus nephritis. Lupus 14: 890-895.

10. Faurschou M, Dreyer L, Kamper AL, http://www.ncbi.nlm. nih.gov/pubmed?term=Starklint $\% 20 \mathrm{H} \% 5 \mathrm{BAuthor} \% 5 \mathrm{D} \& \mathrm{c}-$ author=true\&cauthor_uid=20191478et al. (2010) Long-term mortality and renal outcome in a cohort of 100 patients with lupus nephritis. Arthritis Care Res (Hoboken) 62: 873-880.

11. Reich HN, Gladman DD, Urowitz MB, et al. (2011): Persistent proteinuria and dyslipidemia increase the risk of progressive chronic kidney disease in lupus erythematosus. Kidney Int 79: 914-920.

12. Resende AL, Titan SM, Barros RT, Woronik V (2011): Worse renal outcome of lupus nephritis in male patients: a case-control study. Lupus 20: 561-567.

13. Moon SJ, Kwok SK, Ju JH, et al. (2011): Predictors of chronic kidney disease in Korean patients with lupus nephritis. J Rheumatol 38: 2588-2597.

14. Guo Q, Lu X, Miao L, et al. (2010): Analysis of clinical manifestations and pathology of lupus nephritis: a retrospective review of 82 cases. Clin Rheumatol 29: 1175-1180.

15. Chong YB, Yap DY, Tang CS, Chan TM (2011): Dyslipidaemia in patients with lupus nephritis. Nephrology (Carlton) 16: 511-517.

16. Yip J, Aghdassi E, Su J, et al. (2010): Serum albumin as a marker for disease activity in patients with systemic lupus erythematosus. J Rheumatol 37: 1667-1672.

17. Hochberg MC (1997): Updating the American College of Rheumatology revised criteria for the classification of systemic lupus erythematosus. Arthritis Rheum 40: 1725.

18. Weening JJ, D'Agati VD, Schwartz MM, et al. (2004) The classification of glomerulonephritis in systemic lupus erythematosus revisited. Kidney Int 65: 521-530.

19. Mosca M, Pasquariello A, Tavoni A, et al. (1997): Predictors of renal outcome in diffuse proliferative glomerulonephritis in systemic lupus erythematosus. Lupus 6: 371-378.

20. Bertsias G, Ioannidis JP, Boletis J, et al. (2008): EULAR recommendations for the management of systemic lupus erythematosus. Report of a Task Force of the EULAR Standing Committee for International Clinical Studies Including Therapeutics. Ann Rheum Dis 67: 195-205.
21. Al Durahim H, Al Ghamdi G, Al Seraya A (2011): Predictors of mortality and end stage renal disease in Saudi patients with lupus nephritis. Lupus 20: 1329-1335.

22. Contreras G, Lenz O, Pardo V, et al. (2006):Outcomes in African Americans and Hispanics with lupus nephritis. Kidney Int 69: 1846-1851.

23. Williams W, Shah D, Sargeant LA (2004): The clinical and epidemiologic features in 140 patients with lupus nephritis in a predominantly black population from one center in Kingston, Jamaica. Am J Med Sci 327: 324-329.

24. Sherer Y, Gorstein A, Fritzler MJ, Shoenfeld Y (2004): Autoantibody explosion in systemic lupus erythematosus: more than 100 different antibodies found in SLE patients. Semin Arthritis Rheum 34: 501-537.

25. Matrat A, Veysseyre-Balter C, Trolliet P, et al. (2011): Simultaneous detection of anti-C1q and anti-double stranded DNA autoantibodies in lupus nephritis: predictive value for renal flares. Lupus 20: 28-34.

26. Hung WT, Chen YM, Lan JL, et al. (2011): Antinucleosome antibodies as a potential biomarker for the evaluation of renal pathological activity in patients with proliferative lupus nephritis. Lupus 20: 1404-1410.

27. Manson JJ, Ma A, Rogers P, et al. (2009): Relationship between anti-dsDNA, anti-nucleosome and anti-alpha-actinin antibodies and markers of renal disease in patients with lupus nephritis: a prospective longitudinal study. Arthritis Res Ther 11: R154.

28. Mosca M, Bencivelli W, Neri R, et al. (2002): Renal flares in 91 SLE patients with diffuse proliferative glomerulonephritis. Kidney Int 61: 1502-1509.

29. Sun HO, Hu WX, Xie HL, et al. (2008): Long-term outcome of Chinese patients with membranous lupus nephropathy. Lupus 17: 56-61. 